\title{
Modified Arthroscopic Latarjet Procedure: Suture- button Fixation will not Cause Obvious Increasement of Superior Instability at 5-Year Follow-up
}

\section{Daqiang Liang}

Shenzhen Second People's Hospital

Haifeng Liu

Shenzhen Second People's Hospital

Xinzhi Liang

Shenzhen Second People's Hospital

Qihuang Qin

Shenzhen Second People's Hospital

Lujue Long

Shenzhen Second People's Hospital

\section{Yong Huang}

Shenzhen Second People's Hospital

Wei Lu

Shenzhen Second People's Hospital

Zhenhan Deng ( $\nabla$ dengzhenhan@email.szu.edu.cn )

Shenzhen Second People's Hospital https://orcid.org/0000-0003-0522-8269

\section{Research}

Keywords: Shoulder dislocation, Latarjet procedure, Acromiaohumeral distance, CT

Posted Date: November 18th, 2020

DOl: https://doi.org/10.21203/rs.3.rs-105908/v1

License: @ (i) This work is licensed under a Creative Commons Attribution 4.0 International License. Read Full License 


\section{Abstract}

Background: Whether coracoacromial ligament (CAL) release during Latarjet procedure will increase superior instability of shoulder joint postoperatively remains controversial. This study aims to observe changes in the acromiaohumeral distance (AHD) of patients who underwent modified double-button Latarjet procedure and provide evidence to address the issue.

Methods: A retrospective analysis was conducted among 155 patients who underwent modified doublebutton Latarjet procedure in our department from 2013 to 2015. Preoperative CT scan of bilateral shoulders were used for glenoid defect evaluation. CT scans were performed immediately after operation (PO 0) and during the follow-up at 6, 36, and 60 months postoperatively (POM) to observe the healing and remodeling of the graft, and AHD was measured. The intact sides were set as control group. VAS and objective shoulder scores, including ASES, ROWE, and Walch-Duplay scores, were recorded at each time point.

Results: A total of 104 cases who met the criteria completed follow up. The average follow-up time was $62.6 \pm 2.4$ months. Compared with preoperative conditions, the function scores of the shoulders were significantly improved at the last follow-up. There were no statistical differences of the AHD values between bilateral shoulders preoperatively. The AHD values at PO 0 and POM 6 were significantly higher than those of intact side $(p<0.05)$. The AHD values at POM 36 and 60 were slightly higher than those of intact side and the differences were statistically significant $(p<0.05)$.

Conclusions: The modified double-button Latarjet procedure not only offers satisfactory therapeutic effect but also will not cause obvious superior instability at the 5-year follow-up.

\section{Background}

Recurrent anterior shoulder dislocation with obvious glenoid defect has always been a clinical concern for shoulder surgeons. Anterior subluxation and dislocation account for $52.1 \%$ of all shoulder instability injuries, and approximately $30 \%$ of which requires surgical treatment[1]. At present, the arthroscopic Latarjet procedure has gradually become popular for the treatment of shoulder dislocation with evident glenoid defect, and many reports have confirmed that Latarjet procedure has excellent clinical results[2,3,4].

Although arthroscopic Latarjet procedure is effective, it has to transfer the coracoid and conjoint tendon, which changes the anatomic relationship and subsequent results. Coracoacromial ligament (CAL) needs to be cut-off during the surgery, while previous studies have confirmed CAL's function on maintaining superior stability of shoulder joint $[5,6]$. This procedure arouses surgeons' concern of subsequent superior instability, which may result in secondary acromial impingement, rotator cuff tear, and even anterosuperior dislocation of the humeral head, leading to osteoarthritis and function limitation. 
Previous studies clarified the important role of the coracoacromial arch, especially the CAL, in maintaining the anterosuperior stability of the glenohumeral joint[7]. CAL can physiologically provide superior stability. Denard et al found that in patients with rotator cuff tear associated with acromial impingement, the humeral head shifted up to varying degrees after acromioplasty and CAL was released[8]. In another cadaveric study, the upward movement of the humeral head was more pronounced when upward axial stress was applied to the shoulder joint specimens after cutting off the CAL. This finding also clarifies that the CAL restricts the upward movement of the humeral head[3]. Several scholars believe that in patients with massive rotator cuff tear, the CAL should be preserved to avoid subsequent anterosuperior instability postoperatively[7].

Our previous works demonstrated excellent outcome with few complications and no degenerative changes during the follow-up of patients who underwent modified suture-button arthroscopic Latarjet procedure[9,10]. In the present study, pre- and postoperative acromiaohumeral distances (AHDs) were measured in patients who underwent Latarjet procedure in our department with 5 years of follow-up to evaluate the effects of the modified procedure on increasing superior instability of shoulder joint postoperatively.

\section{Materials And Methods}

\subsection{Subjects}

This retrospective study was approved by the hospital ethics committee of the First Affiliated Hospital of Shenzhen University, Shenzhen Second People's Hospital (reference no. 20190223), and all patients gave informed consent preoperatively. From October 2013 to October 2015, 155 patients underwent modified arthroscopic Latarjet procedure with double-button fixation in our department and 104 of them met the following inclusion criteria: (1) over $20 \%$ of glenoid defect, (2) over $15 \%$ of glenoid defect combined with an Instability Severity Index Score of higher than 6, (3) 10\% to 15\% of glenoid defect in contact sport athletes, (4) previous Bankart repair failure, (5) no multi-direction laxity, (6) no evident rotator cuff tear signs and imaging findings before surgery, and (7) over 5 years of follow-up. The exclusion criteria are as follows: (1) epilepsy, (2) cannot complete follow-up or incomplete follow up data, and (3) previous shoulder surgery history except Bankart repair.

\subsection{Operative Techniques}

All surgeries were performed by a senior chief physician (WL). The modified Latarjet surgical technique was mentioned in our previous publications $[9,10,11]$. Briefly, this modified procedure included three basic steps, as follows. (1) The coracoid bone graft and conjoint tendon were prepared through a $2.5 \mathrm{~cm}$ miniopen incision, starting from $1 \mathrm{~cm}$ below the coracoid process in the direction of the axilla. The CAL and part of the pectoralis minor muscle were cut $1 \mathrm{~cm}$ away from the border of the coracoid. The osteotomy of the coracoid process was then performed at its bend using an oscillating saw to ensure graft of 20 $\mathrm{mm}$ length. Two bone tunnels were drilled with a distance of $6 \mathrm{~mm}$ in the bone graft along its axis. Highstrength sutures (Ultrabraid \#2 white suture assembly; Smith \& Nephew) were pulled into the distal 
tunnel. Three high-strength sutures were pulled into the central hole of a suture button (Endobutton; Smith \& Nephew) and then through the proximal bone tunnel. (2) The anterior (including a part of the incision used for graft acquirement), standard anterolateral, and posterior portals were prepared. The glenoid was marked at the 4 o'clock position, and then the subscapularis muscle was split from back to front until the anterior fascia became visible. A switch stick was used to protect the axillary nerve from damage. The muscle was split with a $1.5 \mathrm{~cm}$ diameter window for bone graft transfer. (3) The glenoid tunnel was drilled where the suture linked to the graft was passed, and the graft was pulled into the glenohumeral joint via the sutures. Then, another endobutton with sutures of the first endobutton going through was prepared and Tennessee knot was set for fixation. A knotless suture anchor for antirotation (PushLock, Arthrex) was fixed to the glenoid.

\subsection{Rehabilitation Protocol}

Standardized rehabilitation protocols were applied. All the patients' arms were immobilized in adduction and internal rotation position in a sling for 6 weeks. Physiotherapy started the day after surgery with pendulum exercises performed several times per day, followed by a 6-week rehabilitation program. During the period, active exercise and workout with weights or pulleys were prohibited. Active exercises with weight, active forward flexion, and passive external rotation would be allowed at 6 weeks postoperatively. Active movement in all directions was initialized at 3 months postoperatively (POM). Active biceps tendon contraction training was started and gradually increased at POM 3. Contact sports or motions with "risks" were not allowed until POM 6.

\subsection{Follow-up}

CT scan of bilateral shoulders were performed preoperatively to evaluate glenoid defect. At immediate postoperatively (PO 0), POM 6, POM 36, and POM 60, the CT scan of the surgical side shoulder was conducted to observe graft position, absorption, remodeling, and graft-glenoid interface healing. Preoperative and postoperative clinical results were assessed using a visual analog scale (VAS) for pain evaluation. American Shoulder and Elbow Surgeons (ASES), ROWE, and Walch-Duplay scores were recorded at each time point for clinical function assessment. Complications that occurred intraoperatively and postoperatively were recorded.

\subsection{Radiological Assessment}

CT scan images were used to measure AHD changes before surgery and during the follow-up. This method had been reported before with high accuracy and reliability[12]. The patient should keep the affected limb relaxed and place it at $0^{\circ}$ abduction and neutral rotation. The images of the oblique coronal view (parallel to the plane of the scapula) on CT was used to measure AHD. To ensure that all cases in the same anatomical position, we performed measurement by choosing the middle slice of all images, in which the glenoid was observed. When the number of images was even, an image with a larger glenoid was chosen for measurement. A horizontal line A was made through the lowest point of the acromion, which was parallel to the lower surface of the acromion, and a horizontal line B parallel to the horizontal 
line A was created through the highest point of the humeral head. The perpendicular distance between $A$ and B was recorded as AHD. (Fig. 1)

\subsection{Statistical Analysis}

The measurement data were expressed as mean $\pm S D$, and the data were analyzed statistically using SPSS 16.0 software (Chicago, IL, U.S.A). Pairwise comparison was performed using paired $t$-test, and $\mathrm{p}<$ 0.05 was considered statistically significant.

\section{Results}

\subsection{Baseline Characteristics}

A total of 155 patients who underwent modified arthroscopic Latarjet procedure were selected. 7 cases with epilepsy, 15 cases without enough follow up time and 29 cases without complete follow up data were excluded. Finally, 104 patients with an average age of $29.6 \pm 7.5$ years were enrolled. The average follow-up time was $62.6 \pm 2.4$ months. At the last follow-up, all patients could return to daily life activities and 90 of them could resume preoperative sports level. No axillary nerves injury and vascular injury occurred in any patient. One 40 years old female patient suffered redislocation because of traffic accident and another 42 years old female patient got shoulder stiffness and recovered after physical therapy. The total complication rate was $1.8 \%$. No one complained about symptoms and signs related to rotator cuff tear and acromial impingement. Among these patients, 66 were male, 38 were female, 60 had injury in the left shoulder, and 44 had injury in the right shoulder. All patients were diagnosed of shoulder dislocation due to trauma or sports activity. The average glenoid defect was $23.4 \% \pm 4.1 \%(17 \%$ to $30 \%)$, and the average time from initial dislocation to surgery was $24.8 \pm 11.2$ months ( $8-44$ months, Table 1 ).

\subsection{Clinical Assessment}

All patients returned to normal daily activities at the last follow-up. At PO 0 , significantly decreased VAS score ( $3.1 \pm 1.3$ vs $1.2 \pm 0.7, p<0.05)$ and increased ASES (73.2 \pm 14.1 vs $94.4 \pm 4.0, p<0.05)$, ROWE score $(41.7 \pm 8.9$ vs $94.5 \pm 2.7, p<0.05)$ and Walch-Duplay scores $(64.4 \pm 9.8$ vs $95.7 \pm 3.5, p<0.05$, Table 2) compared with the preoperative conditions, thereby indicating evident pain relief and the improvement of the function of the injury shoulder.

\subsection{AHD Measurement}

The AHD of intact side was $7.8 \pm 0.8 \mathrm{~mm}$ and set as control group. The preoperative AHD of affected side was $7.8 \pm 0.8 \mathrm{~mm}$ and there was no statistical difference compared to intact side $(p>0.05)$. The AHD at PO $0(9.6 \pm 0.7 \mathrm{~mm})$ significantly increased compared to control group $(p<0.05)$. During the follow-up, the AHD at POM 6 was $8.6 \pm 0.9 \mathrm{~mm}$, which was also higher than control group $(\mathrm{p}<0.05)$. The AHD values at POM 36 and 60 were $8.0 \pm 0.8$ and $8.1 \pm 0.8 \mathrm{~mm}$ respectively, which were slightly higher than the control group and with statistical significance $(p<0.05$, Table 3$)$. Fig. 2 shows AHD measurement at full followup in one representative case. 


\section{Discussion}

To the best of our knowledge, this study is the first to observe changes in AHD after Latarjet procedure by evaluating whether the CAL resection of the Latarjet procedure will increase superior instability postoperatively. AHD was significantly improved immediately after Latarjet procedure with a trend of narrow during the follow-up. Our study found that the modified double-button Latarjet procedure was effective in treating recurrent shoulder dislocation without evident increasement of superior instability of shoulder joint at over 5 years of follow-up. This result may help eliminate concerns about post-operative superior instability, even subsequent acromial impingement, rotator cuff tear and superior dislocation caused by the damage of CAL.

According to clinical and biomechanical studies mentioned above, Latarjet procedure should lead to the possibility of superior instability of the glenohumeral joint when performing CAL release and coracoid transfer $[5,6,13,14]$. However, extremely few studies have focused on subsequent effects caused by CAL resection during Latarjet procedure. Several cadaveric studies observed that humeral head moves upward to varying degrees after CAL release and coracoid transfer. However, the frozen specimens that could not accurately reflect the physiological situation of the clinical patients. Aurich et al used congruent-arc Latarjet procedure to treat patients with recurrent shoulder dislocation[15]. To prevent postoperative superior instability of shoulder joint, they used pectoralis minor fascial flap to perform a one-stage reconstruction of the CAL. None of the participants had postoperative complications and no secondary superior instability at 1 year of follow-up. However, the sample number was small with six cases applied and lacked comparison with the effect of traditional Latarjet procedure. Therefore, we cannot clarify the specific changes in the humeral head movement of clinical patients at different periods after traditional Latarjet procedure. At present, clinical studies that focused on superior instability after Latarjet procedure have not been conducted yet.

We measured the AHD on CT scan images to reflect superior instability of shoulder joint after Latarjet procedure. CT, ultrasound, X-ray, and ultrasound have certain reliability in measuring AHD. CT have better reliability and accuracy than $\mathrm{X}$-ray and can be used as a tool in assessing bone graft healing and absorption at the same time[16]. Regardless of various applications, such as evaluation post-reverse shoulder replacement, rotator cuff tear risk assessment, or athletic evaluation, the ultrasound measurement of AHD has high reliability $[17,18,19]$. Although ultrasound is simple and repeatable without radiation, it depends on operating level and patient's cooperation to a large extent. In the present study, the AHD measurement was completed under a standard method to avoid subjective deviation.

The release of the CAL will decrease the stability between the humeral head and acromion at the zero time, so that the acromion would move upward. Given the tension and depressive effects of the transferred bone graft and conjoint tendon on the muscle fibers of the lower half of the subscapularis, this effect was transmitted to the humeral head and at last caused AHD increasement (Fig. 3). According to our previous studies $[9,10]$, patients received our modified arthroscopic Latarjet procedure would get complete bone healing at about POM 6 and the bone graft kept remodeling up to more than 3 years. 
Therefore we set the follow up time period as 6 months, 3 years and 5 years. During the follow-up, the gradually decreased AHD may be related to the reformation of the ligament-like structure connecting the conjoint tendon and acromion and the readaptation of the glenohumeral joint caused by changes in the patient's own muscle strength[20]. That could be considered as compensatory of CAL function and recovery of superior instability. Nonetheless, no case with narrower AHD after 5 years of follow-up than that before surgery was observed.

The limitations of this study should be acknowledged. First, this work adopted a retrospective design without the setup of the control groups treated with other surgical method, such as Bankart, Bristow, et al. Second, the CT images in this study were all scanned on relaxed state, and we did not obtain shoulder images under axial upward stress. Therefore, the conclusion cannot accurately completely reflect the various mechanics of the patient after resuming exercise. Future directions require kinematic experiments to observe the specific humeral head movement of patients during different activity after Latarjet procedure, compare clinical outcomes with other arthroscopic surgeries, and related biomechanical studies will be conducted to enhance our results.

\section{Conclusion}

The modified double-button Latarjet procedure not only offers satisfactory therapeutic effect but also will not cause obvious superior instability at 5-year follow-up. This result may help eliminate concerns about increased superior instability and subsequent complications after Latarjet procedure.

\section{Declarations}

\section{Ethics approval and consent to participate}

This study was approved by the hospital ethics committee of the First Affiliated Hospital of Shenzhen University, Shenzhen Second People's Hospital (reference no. 20190223), and all patients gave informed consent preoperatively.

\section{Consent for publication}

Not applicable.

\section{Availability of data and material}

The datasets analyzed during the current study are available from the corresponding author upon reasonable request.

\section{Competing interests}

The authors declare that they have no competing interests. 


\section{Funding}

This work was supported by supported by the National Natural Science Foundation of China (81902303), Guangdong Basic and Applied Basic Research Foundation (2020A151501048), Shenzhen Science and Technology Project (JCYJ20190806164216661), and Clinical Research Project of Shezhen Second People's Hospital (20203357028).

\section{Authors' contributions}

Study design: ZD, WL. Drafting manuscript: ZD, LD. Data collection: ZD, DL, HL. Data analysis: ZD, XL, QQ, $\mathrm{LL}, \mathrm{YH}$. The author (s) read and approved the final manuscript.

\section{Acknowledgements}

None.

\section{References}

1. Trojan JD, Meyer LE, Edgar CM, BrownSM, MulcaheyMK. Epidemiology of Shoulder Instability Injuries in Collision Collegiate Sports From 2009 to 2014. Arthroscopy. 2020;36:36-43.

2. Ali J, Altintas B, Pulatkan A, BoykinRE, AksoyDO, Bilsel K. Open Versus Arthroscopic Latarjet Procedure for the Treatment of Chronic Anterior Glenohumeral Instability With Glenoid Bone Loss. Arthroscopy. 2020;36:940-9.

3. Hardy A, Sabatier V, Laboudie P, SchochB, Nourissat G, Valenti P, et al. Outcomes After Latarjet Procedure: Patients With First-Time Versus Recurrent Dislocations. Am J Sports Med. 2020;48:21-6.

4. Hurley ET, Montgomery C, Jamal MS, ShimozonoY, AliZ, Pauzenberger L, et al. Return to Play After the Latarjet Procedure for Anterior Shoulder Instability: A Systematic Review. Am J Sports Med. 2019;47:3002-8.

5. Lee TQ, Black AD, Tibone JE, McMahon PJ. Release of the coracoacromial ligament can lead to glenohumeral laxity: a biomechanical study. J Shoulder Elbow Surg. 2001;10:68-72.

6. Wellmann M, Petersen W, Zantop T, Schanz S, Raschke MJ, Hurschler C. Effect of coracoacromial ligament resection on glenohumeral stability under active muscle loading in an in vitro model. Arthroscopy. 2008;24:1258-64.

7. Su WR, Budoff JE, Luo ZP. The effect of coracoacromial ligament excision and acromioplasty on superior and anterosuperior glenohumeral stability. Arthroscopy. 2009;25:13-8.

8. Denard PJ, Bahney TJ, Kirby SB, Orfaly RM. Contact pressure and glenohumeral translation following subacromial decompression: how much is enough? Orthopedics. 2010;33:805.

9. Xu J, Liu H, Lu W, DengZH, ZhuWM, Peng LQ, et al. Modified Arthroscopic Latarjet Procedure: SutureButton Fixation Achieves Excellent Remodeling at 3-Year Follow-up. Am J Sports Med. 2020;48:3947. 
10. Xu J, Liu H, Lu W, ZhuWM, PengLQ, Ouyang K, et al. Clinical outcomes and radiologic assessment of a modified suture button arthroscopic Latarjet procedure. BMC Musculoskelet Disord. 2019;20:173.

11. Boileau P, Saliken D, Gendre P, SeetoBL, d'OllonneT, Gonzalez JF, et al. Arthroscopic Latarjet: SutureButton Fixation Is a Safe and Reliable Alternative to Screw Fixation. Arthroscopy. 2019;35:1050-61.

12. Werner CM, Conrad SJ, Meyer DC, Keller A, Hodler J, Gerber C. Intermethod agreement and interobserver correlation of radiologic acromiohumeral distance measurements. J Shoulder Elbow Surg. 2008;17:237-40.

13. Degen RM, Giles JW, Boons HW, Litchfield RB, Johnson JA, Athwal GS. A biomechanical assessment of superior shoulder translation after reconstruction of anterior glenoid bone defects: The Latarjet procedure versus allograft reconstruction. Int J Shoulder Surg. 2013;7:7-13.

14. Hockman DE, Lucas GL, Roth $\mathrm{CA}$. Role of the coracoacromial ligament as restraint after shoulder hemiarthroplasty. Clin Orthop Relat Res. 2004:80-2.

15. Aurich M, Hofmann GO, Gras F. Reconstruction of the coracoacromial ligament during a modified Latarjet procedure: a case series. BMC Musculoskelet Disord. 2015;16:238.

16. McCreesh KM, Crotty JM, Lewis JS. Acromiohumeral distance measurement in rotator cuff tendinopathy: is there a reliable, clinically applicable method? A systematic review. Br J Sports Med. 2015;49:298-305.

17. Mackenzie TA, Bdaiwi AH, Herrington L, Cools A. Inter-rater Reliability of Real-Time Ultrasound to Measure Acromiohumeral Distance. PM R. 2016;8:629-34.

18. McCreesh KM, Anjum S, Crotty JM, Lewis JS. Ultrasound measures of supraspinatus tendon thickness and acromiohumeral distance in rotator cuff tendinopathy are reliable. J Clin Ultrasound. 2016;44:159-66.

19. Werner BS, Jacquot A, Mole D, Walch G. Is radiographic measurement of acromiohumeral distance on anteroposterior view after reverse shoulder arthroplasty reliable? J Shoulder Elbow Surg. 2016;25:e276-80.

20. Smolen D, Went P, Tomala D, SternbergC, LafosseL, Leuzinger J. Identification of a Remodeled Neotendon After Arthroscopic Latarjet Procedure. Arthroscopy. 2017;33:534-42.

\section{Tables}




\begin{tabular}{|ll|}
\hline Table 1 Patient Data & \\
\hline Parameter & Value \\
\hline Age, mean \pm SD (range), y & $29.6 \pm 7.5(19-45)$ \\
\hline Gender,male/female, $\mathrm{n}$ & $66 / 38$ \\
\hline Side, left/right, $\mathrm{n}$ & $60 / 44$ \\
\hline Number of dislocations & $8.4 \pm 4.7$ \\
\hline Body mass index, kg/m2 & $23.2 \pm 4.3$ \\
\hline Glenoid defect area, \% & $23.4 \pm 4.1$ \\
\hline$>20 \%, \mathrm{n}$ & 60 \\
\hline $15 \%-20 \%, \mathrm{n}$ & 26 \\
\hline $10 \%-14 \%, \mathrm{n}$ & 12 \\
\hline Bankart failure, $\mathrm{n}$ & 6 \\
\hline Hill-Sachs injury, $\mathrm{n}$ & 100 \\
\hline
\end{tabular}

\begin{tabular}{|llll|}
\hline \multicolumn{4}{l}{ Table 2 Functional results at preoperative and final follow-up } \\
Parameter & Preoperative & Final follow-up & p-Value \\
VAS for pain (during motion) & $3.1 \pm 1.3$ & $1.2 \pm 0.7$ & $<0.001$ \\
\hline ASES score & $73.2 \pm 14.1$ & $94.4 \pm 4.0$ & $<0.001$ \\
ROWE score & $41.7 \pm 8.9$ & $94.5 \pm 2.7$ & $<0.001$ \\
Walch-Duplay score & $64.4 \pm 9.8$ & $95.7 \pm 3.5$ & $<0.001$ \\
\hline
\end{tabular}




\begin{tabular}{|lll|}
\hline \multicolumn{2}{|l|}{ Table 3 AHD Value at Different Follow-up Period } & \\
\hline Intact side AHD (control group) & Affected side AHD & P Value \\
\hline $7.8 \pm 0.8$ (Preoperative) & $7.8 \pm 0.8$ (Preoperative) & 0.851 \\
\hline & $9.6 \pm 0.7$ (POM 0) & $<.001$ \\
\hline $8.6 \pm 0.9(\mathrm{POM} 6)$ & $<.001$ \\
\hline $8.0 \pm 0.8(\mathrm{POM} 36)$ & $<.001$ \\
\hline $7.9 \pm 0.8(\mathrm{POM} 60)$ & $<.001$ \\
\hline
\end{tabular}

Abbreviation: $\mathrm{AHD}=$ acromiaohumeral distances; $\mathrm{POM}=$ postoperative months

\section{Figures}

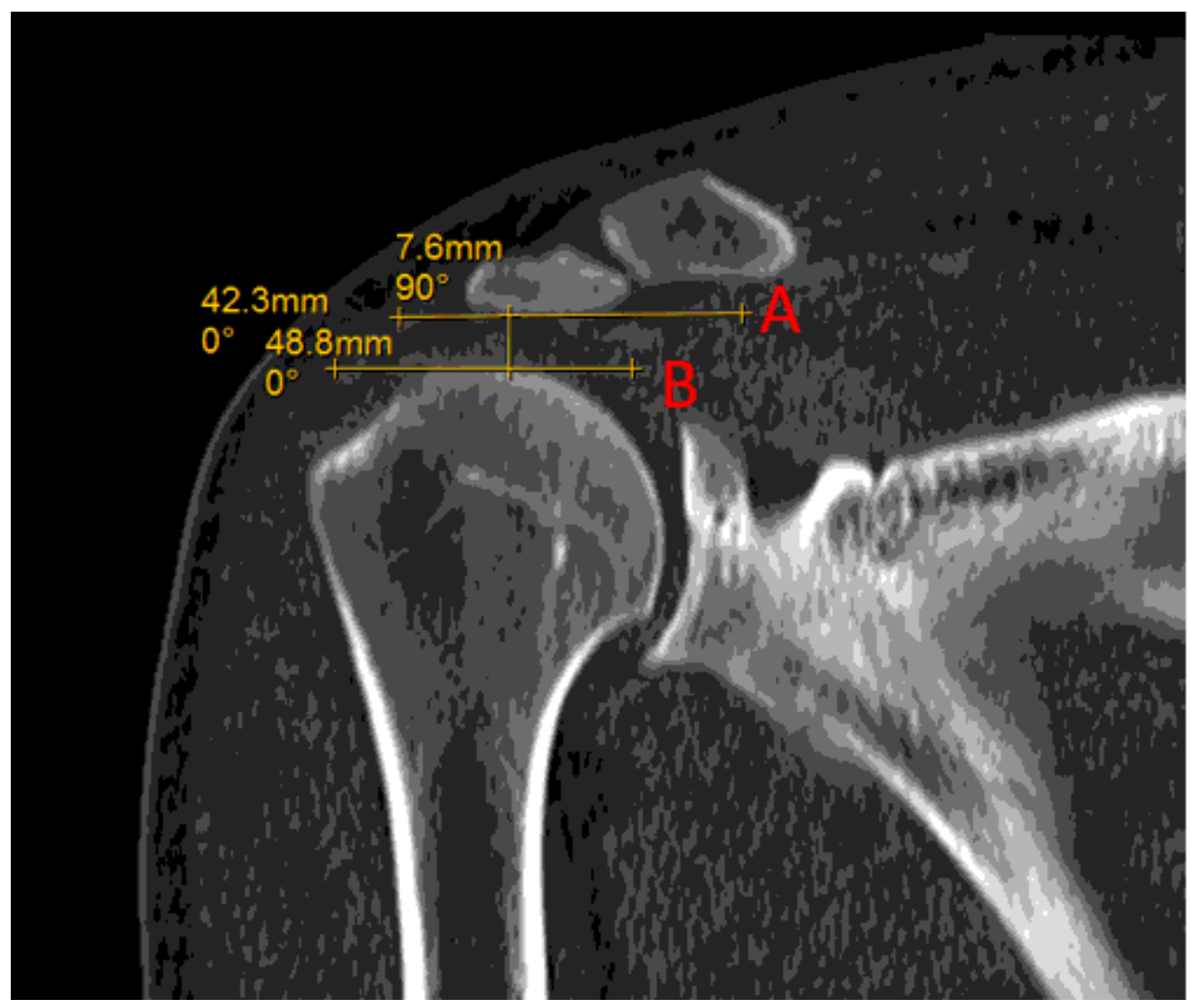

\section{Figure 1}

Strategy of AHD measurement of coronal view on CT scan image. 


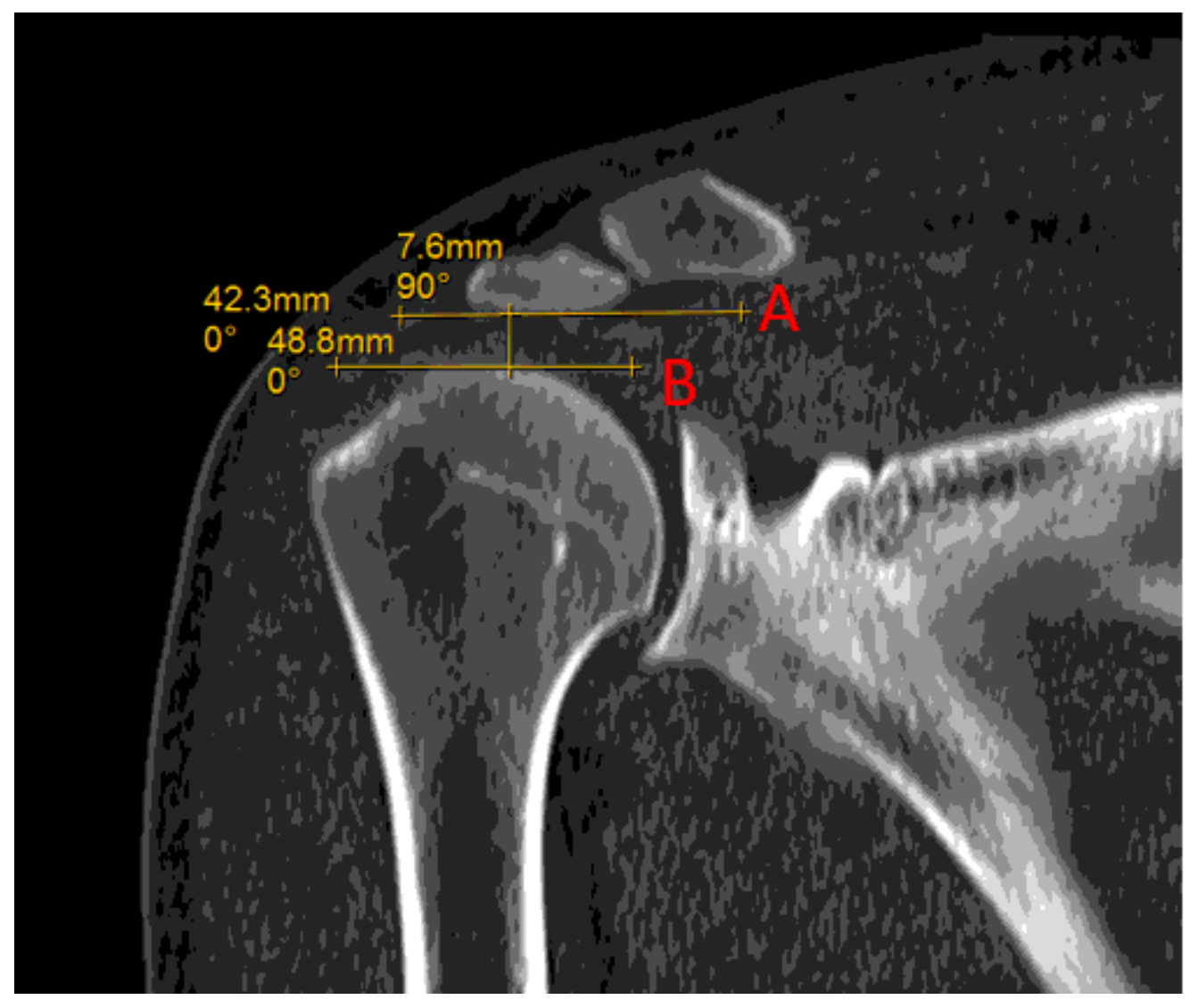

Figure 1

Strategy of AHD measurement of coronal view on CT scan image. 


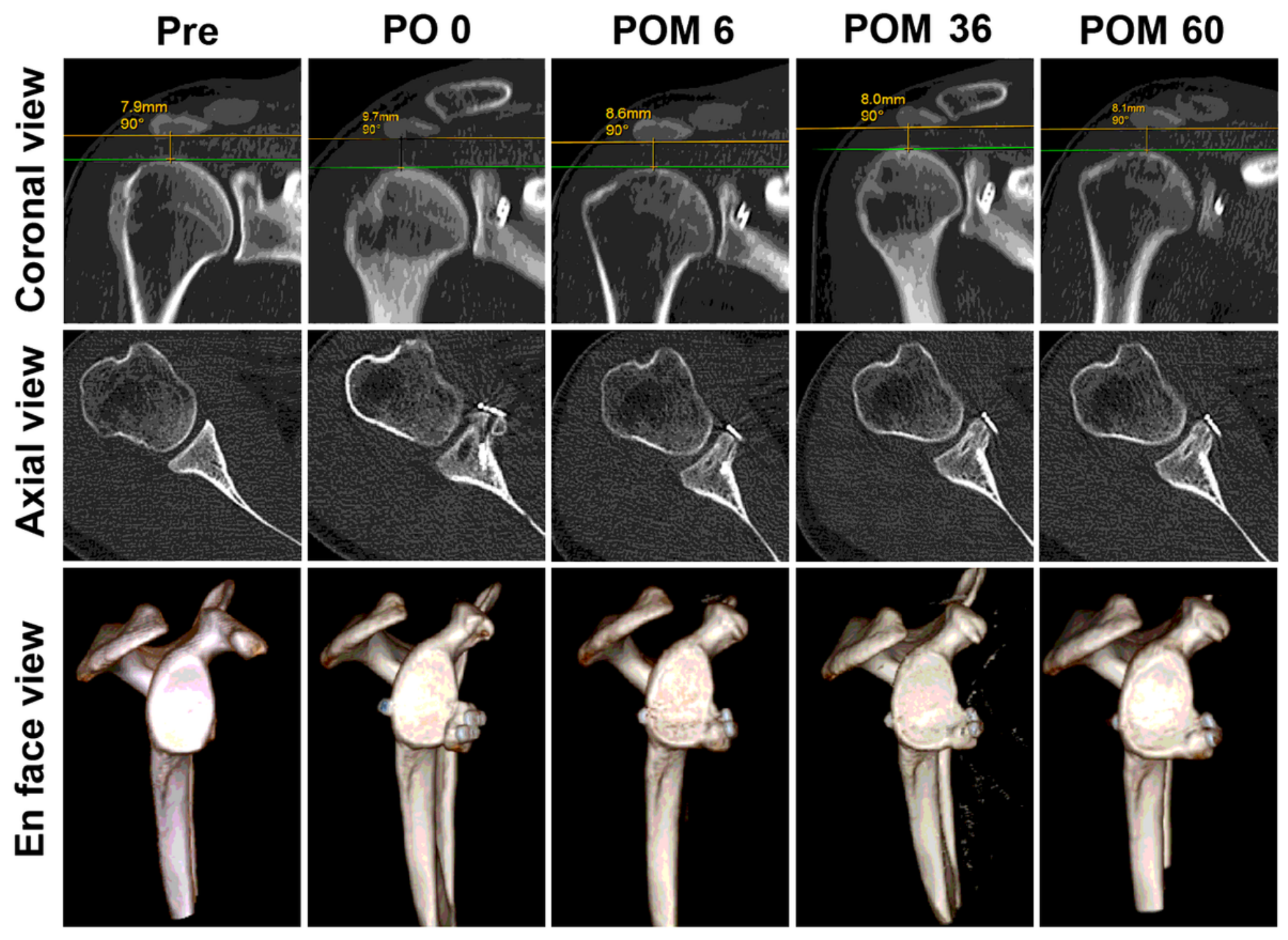

Figure 2

AHD measurement (coronal view), observation of graft position, remodeling, and graft-glenoid interface healing (axial and en face view) of 3D reconstructed CT scan images at different time points from one representative case that underwent Latarjet procedure. Pre, preoperative; PO 0, immediate after operation; POM: month postoperatively. 


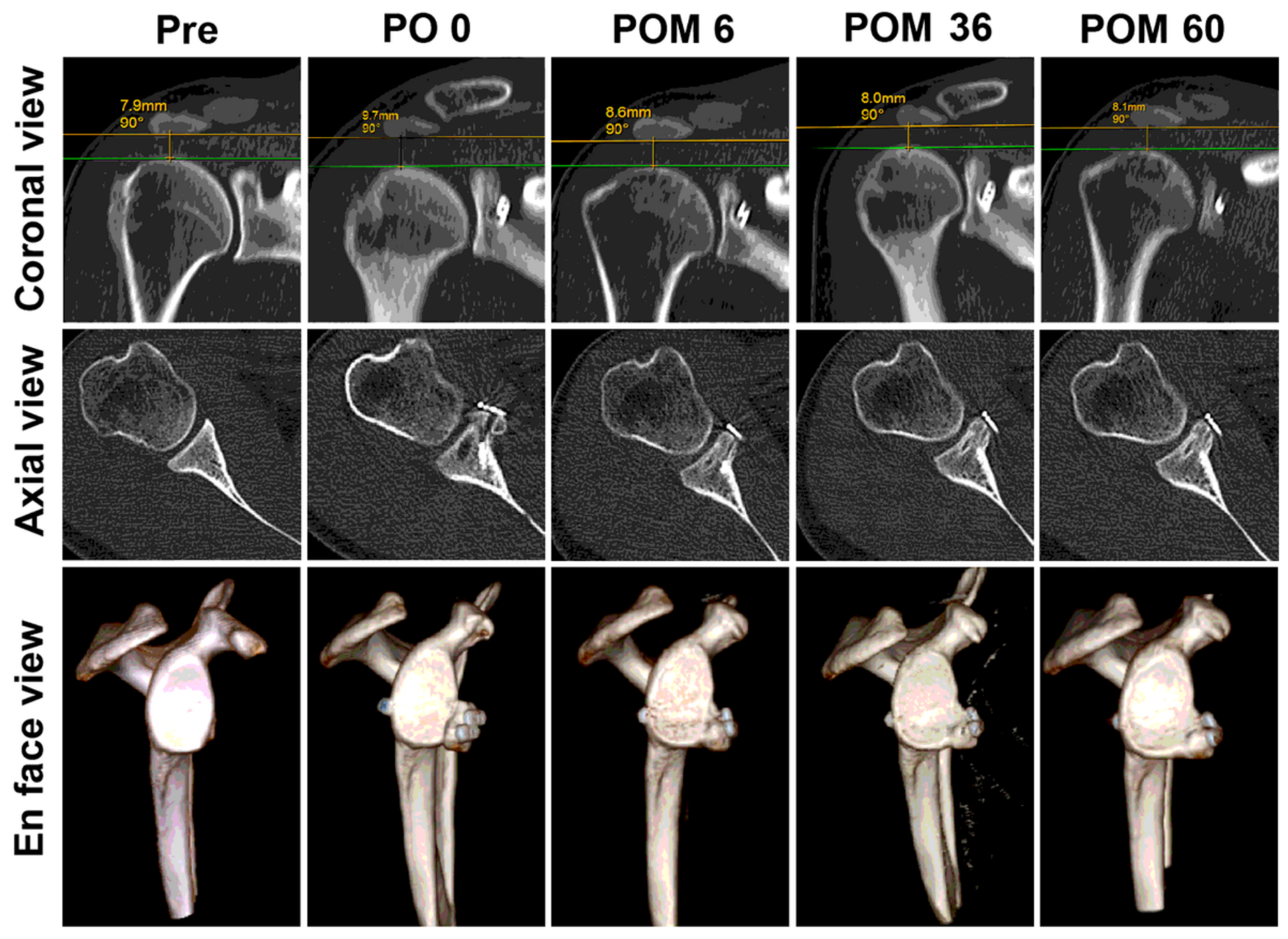

Figure 2

AHD measurement (coronal view), observation of graft position, remodeling, and graft-glenoid interface healing (axial and en face view) of 3D reconstructed CT scan images at different time points from one representative case that underwent Latarjet procedure. Pre, preoperative; PO 0 , immediate after operation; POM: month postoperatively. 


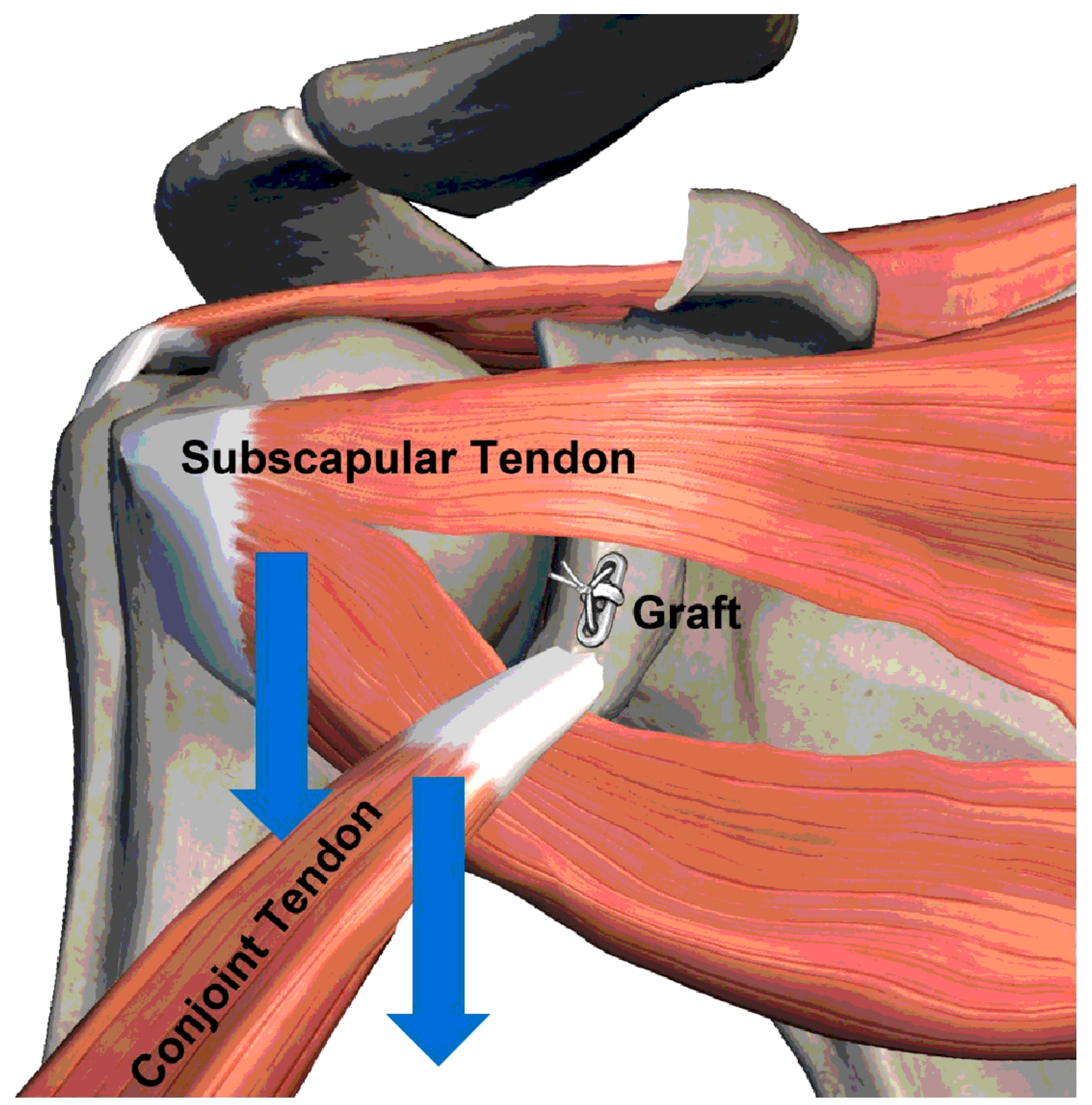

Figure 3

Depressive effect that subscapular and conjoint tendons on the humeral head. 


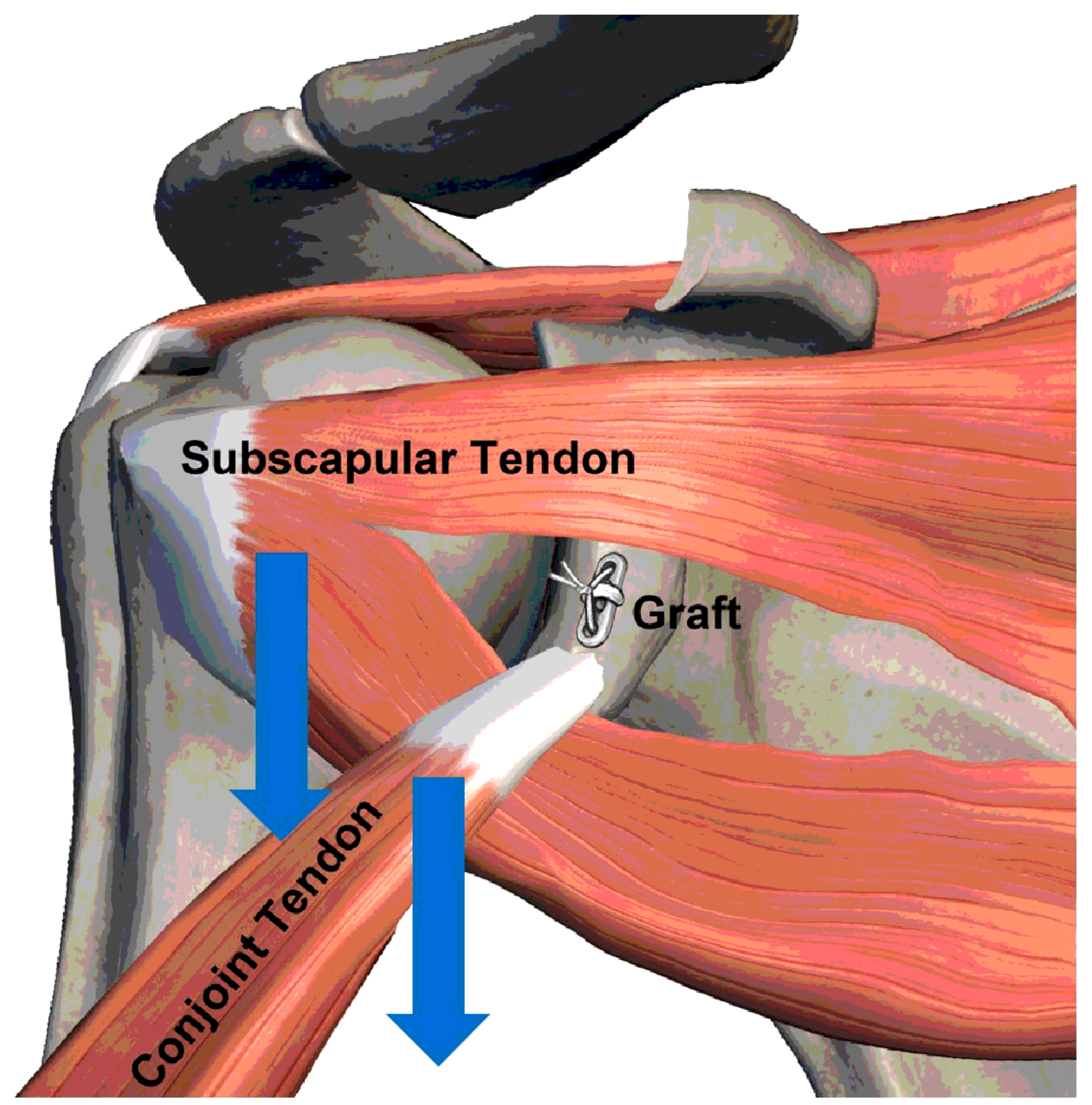

Figure 3

Depressive effect that subscapular and conjoint tendons on the humeral head.

\section{Supplementary Files}

This is a list of supplementary files associated with this preprint. Click to download.

- SupplementaryfileManuscriptpdf.pdf

- SupplementaryfileManuscriptpdf.pdf 